\title{
Advanced endoscopic features of ulcerative colitis-associated neoplasias: Quantification of autofluorescence imaging
}

\author{
SHINICHIRO YOSHIOKA ${ }^{1}$, KEIICHI MITSUYAMA ${ }^{1,2}$, HIDETOSHI TAKEDATSU $^{1}$, KOTARO KUWAKI $^{1}$, \\ RYOSUKE YAMAUCHI $^{1}$, HIROSHI YAMASAKI ${ }^{1}$, SHUHEI FUKUNAGA ${ }^{1}$, JUN AKIBA ${ }^{3}$, \\ TETSUSHI KINUGASA ${ }^{4}$, YOSHITO AKAGI ${ }^{4}$, OSAMU TSURUTA ${ }^{1}$ and TAKUJI TORIMURA ${ }^{1}$ \\ ${ }^{1}$ Division of Gastroenterology, Department of Medicine, Kurume University School of Medicine; \\ ${ }^{2}$ Inflammatory Bowel Disease Center, Kurume University School of Medicine; Departments of \\ ${ }^{3}$ Pathology, ${ }^{4}$ Surgery, Kurume University School of Medicine, Kurume 830-0011, Japan
}

Received September 24, 2015; Accepted November 5, 2015

DOI: $10.3892 /$ ijo.2015.3284

\begin{abstract}
Ulcerative colitis (UC) patients are well known to carry a higher risk of developing colorectal dysplasia/cancer. However, it is hard to detect the lesion in the early phase during colonoscopy. This pilot study was conducted to analyze the endoscopic characteristics of neoplastic lesions associated with UC using advanced imaging techniques. This is a retrospective analysis of 15 colorectal neoplastic lesion obtained from 11 UC patients during remission who underwent whitelight- and advanced endoscopic imaging techniques, including chromoendoscopy, narrow-band imaging and autofluorescence imaging (AFI), and were treated with surgery. These lesions were analyzed for histology, location, size, shape, color and endoscopic features. The green/red ratio was also assessed to quantify the AFI intensity. All 11 patients had extensive colitis with the median disease duration of 14.0 years. A total of 15 lesions, consisting of 8 high-grade dysplasia and 7 cancer, was mostly located in the distal colon $(86.7 \%, 13 / 15)$ with the mean size of $8.6 \mathrm{~mm}$. The shape was protruding in $46.7 \%$ $(7 / 15)$, flat elevated in $40.0 \%(6 / 15)$ and flat in $13.3 \%(2 / 15)$ and the color was red in $60.0 \%(9 / 15)$, same colored in $33.3 \%$ $(5 / 15)$ and discolored in $6.7 \%(1 / 15)$. The lesion predominantly showed Kudo's neoplastic pit pattern in $86.7 \%(13 / 15$; 5 type IIIL, 7 type IV and 1 type VI) on chromoendoscopy and Sano's neoplastic capillary pattern (type IIIa) in $63.6 \%$ (7/11) on narrow-band imaging, but were colored purple as neoplastic lesions in only $37.5 \%$ (3/8) on AFI. Of note, the AFI green/red ratio was significantly lower in the neoplastic lesions
\end{abstract}

Correspondence to: Dr Keiichi Mitsuyama, Inflammatory Bowel Disease Center, Division of Gastroenterology, Department of Medicine, Kurume University School of Medicine, 67 Asahi-machi, Kurume 830-0011, Japan

E-mail: ibd@med.kurume-u.ac.jp

Key words: ulcerative colitis, colorectal cancer, autofluorescence imaging than UC-involved areas ( $\mathrm{p}=0.00014)$ and UC-uninvolved areas $(p=0.00651)$ irrespective of the lesion's size and histological type. In conclusion, endoscopic analysis based on advanced imaging, in particular AFI quantitation, may be helpful to detect early stage neoplastic lesions in long standing UC. Large-scale, prospective studies are needed.

\section{Introduction}

Ulcerative colitis (UC) patients are well known to carry a higher risk of developing colorectal dysplasia and cancer. These lesions develop from chronic inflamed mucosa and progress through dysplasia to adenocarcinoma, termed the 'inflammation-dysplasia-carcinoma sequence' $(1,2)$. In clinical settings, early detection of the neoplasia is the key to improving the prognosis just as in most cancers. However, UC-associated neoplasias often develop in flat or mildly elevated lesions and are distributed multifocally within an area of intestinal inflammation, making it hard to detect them in early phase during colonoscopy (3-5).

The benefit of neoplasia surveillance colonoscopy in UC has been established. At present, the recommended surveillance strategy involves frequent random biopsies aimed at detecting dysplasia/cancer (6-9). However, this method is limited by sampling error, requires considerable time and cost, and has resulted in only a modest reduction in cancer incidence and mortality (10). Previously, the use of a targeting biopsy on conventional white light imaging (WLI), in which tissue specimens are obtained only when endoscopic findings indicate the possibility of neoplasia, thereby yielding a smaller number of samples, has been proposed $(11,12)$. However, it is still controversial whether a targeted biopsy should replace a random biopsy.

Several advanced endoscopic imaging techniques, such as chromoendoscopy (CE) with dye-spraying (13-15), narrowband imaging (NBI) $(16,17)$ and autofluorescence imaging (AFI) $(18,19)$, which provide a more detailed visualization of the mucosa by enhancing morphology and vascularization, have been developed to improve upon the accuracy afforded by conventional WLI. In sporadic colorectal tumors, it has been clearly shown that these imaging techniques facilitate 
early detection allowing for the removal of the lesions, thus avoiding the need for surgery (13). Advanced endoscopic imaging may also provide better definition and delineation of early stage neoplastic lesions even in UC, increased yield of detection and a decrease in the number of biopsies taken. However, little is known about the role of these techniques in UC-associated neoplastic lesions (20-22).

This pilot study was conducted to analyze the endoscopic characteristics of neoplastic lesions associated with UC using advanced endoscopic imaging techniques. In particular, we validated the role of AFI quantification for the first time.

\section{Patients and methods}

Patient selection and study design. A total of 11 patients (7 men and 4 women; median age, 63 years) who underwent total colonoscopy at Kurume University Hospital between April 2003 and March 2014, who had one or more UC-associated neoplastic lesions that satisfied all of the following inclusion criteria for the study, were enrolled: i) Clinically and histologically diagnosed as having UC; ii) Mucosal status was in remission stage $(0$ or 1 point in the Mayo endoscopic score) because histopathological distinction between inflammation and neoplasia can be extremely difficult in active inflammation; iii) Lesions which were subsequently managed by surgical resection; iv) Lesions in which histopathological evaluation was possible; and v) Lesions which could be excluded as being advanced colorectal cancer. Table I summarized the characteristics of the UC patients studied. The ethics committee of our hospital approved the study protocol and written informed consent was obtained from each of the study participants.

Endoscopic procedure. All patients underwent preparation for colonoscopy by ingesting 2 liters of polyethylene glycolelectrolyte solution on the morning of the procedure. In some cases, scopolamine butylbromide $(10 \mathrm{mg})$ was administered intravenously to avoid bowel movements prior to the examination in those patients in whom this agent was not contraindicated.

Colonoscopy was performed using WLI and AFI of the colon by experienced colonoscopists. The AFI system (Olympus Medical Systems, Tokyo, Japan) used in this study consisted of a light-source system (CLV-260SL), a processor (CV-260SL), a liquid-crystal display monitor, and a specialized video endoscope for AFI detection (CF-FH260AZI). When colorectal lesions were detected, they were observed by switching to the NBI mode by the press of a button in the control head of the endoscope. Subsequently, the lesions were observed by CE using $0.4 \%$ indigo carmine and the WLI mode. After washing the indigo carmine with water insufflation, realtime color analysis on the AFI images was conducted using a personal computer with software for color analysis connected to the endoscopy system. Finally, magnifying endoscopy with NBI and CE using crystal violet was performed to estimate the detail of the encountered lesion.

Conventional endoscopic analysis. Conventional endoscopic features were classified based on size, shape, location and color $(18,23)$.
Table I. Characteristics of the patients studied.

\begin{tabular}{lc}
\hline Characteristics & Data \\
\hline Total number of patients & 11 \\
Gender, male/female & $7 / 4$ \\
Age, years (median, range) & $63(33-74)$ \\
Disease duration, years (median, range) & $14(3-32)$ \\
Area involved & \\
Extensive & 11 \\
Left-sided & 0 \\
Mayo score & \\
Endoscopy & $1(0-2)$ \\
Total & $2(0-8)$ \\
Treatments & \\
None & 0 \\
5-aminosalicylic acid & 8 \\
Prednisolone & 8 \\
Immunomodulators & 2 \\
Anti-tumor necrosis factor & 0 \\
Complication of primary sclerosing cholangitis & 0 \\
\hline
\end{tabular}

Chromoendoscopic analysis. Using magnifying CE, pit patterns were classified into types I through $\mathrm{V}$ based on the classification of Kudo et al $(24,25)$ Type I represents regular round crypts, type II represents stellar or papillary crypts, type III represents small tubular or roundish crypts $\left(\mathrm{III}_{\mathrm{S}}\right)$ or large tubular or roundish crypts $\left(\mathrm{III}_{\mathrm{L}}\right)$, type IV consists of branch- or gyrus-like crypts, and type $\mathrm{V}$ consists of irregular crypts $\left(\mathrm{V}_{\mathrm{I}}\right)$ or non-structural crypts $\left(\mathrm{V}_{\mathrm{N}}\right)$. Type I and II lesions are mostly non-neoplastic, whereas type III, IV and V lesions are mostly neoplastic.

Narrow-band imaging analysis. Using NBI, the lesion was classified using the Sano capillary pattern classification $(26,27)$, with type I (faintly visible micro-vessels surrounding the pits) representing a non-neoplasm, type II (elongated and increased thicker vessels surrounding the pits) representing adenoma, and type III (type IIIa, increased thick vessels unevenly sized with branching and curtailed irregularity; type IIIb, nearly avascular or loose vessels with fragmentation) indicating the detection of cancer.

Autofluorescence imaging analysis. The lesion was assessed for color on AFI $(18,19)$. The color of mucosa was divided into green as non-neoplastic or purple as neoplastic. Due to individual variations in the AFI intensity, the AFI intensity of any identified lesion was also compared with the intensity of the non-neoplastic area (both UC-involved and -uninvolved area) in each individual, and the corrected value, termed green/red $(\mathrm{G} / \mathrm{R})$ ratio, was estimated by dividing the green color tone intensity values by the red color tone intensity values, based on the method previously reported (28). Color tone intensity analysis was carried out with still images of the colorectal 


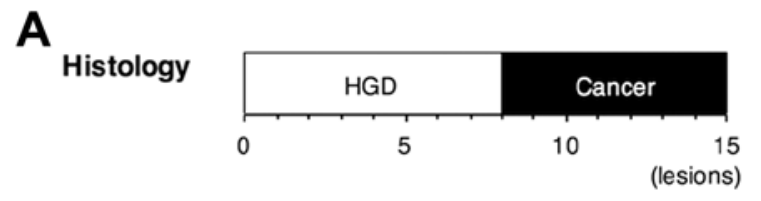

B

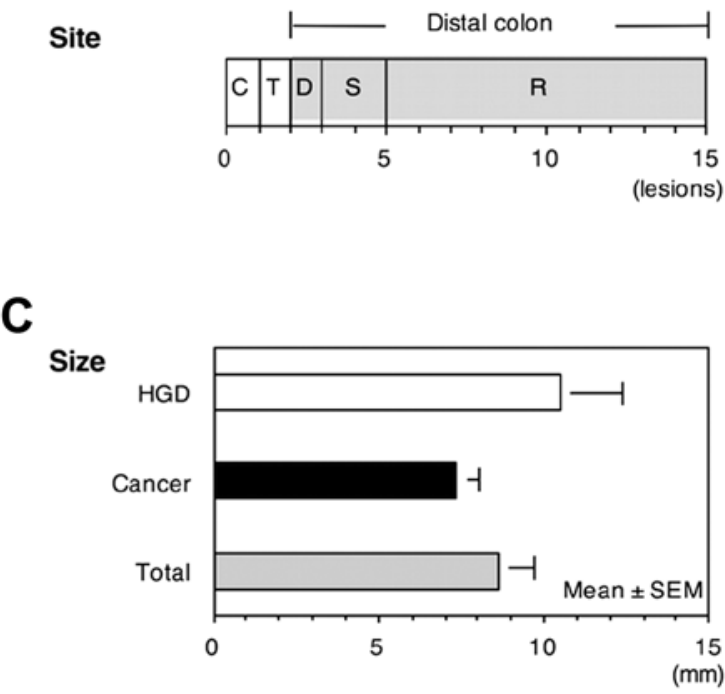

D Shape

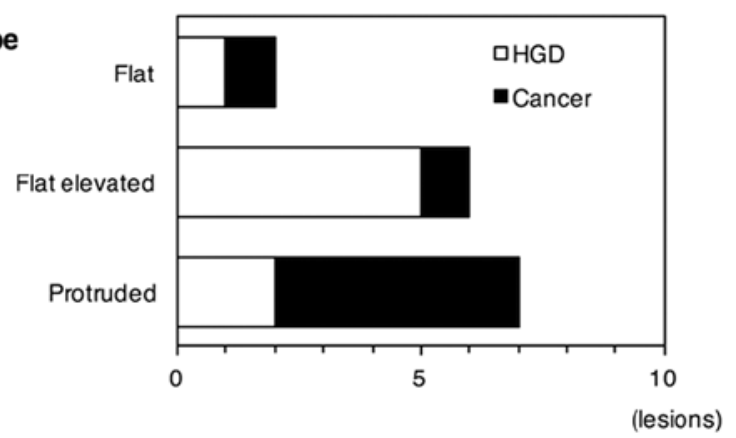

E Color

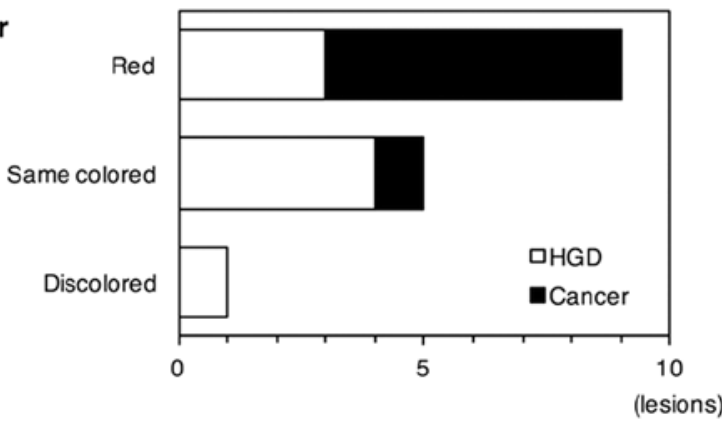

Figure 1. Clinicopathological features of colorectal neoplasia associated with UC according to the histology (A), site (B), size (C), shape (D) and color (E). HGD, high-grade dysplasia. C, cecum; T, transverse colon; D, descending colon; S, sigmoid colon; R, rectum.

lesions using software developed by Rasband, WS, Image J, U.S. National Institutes of Health, Bethesda, MD, USA, http:// rsb.info.nih.gov/ij/, 1997-2012.

Histopathology. Surgically resected specimens were immediately fixed in $10 \%$ buffered formalin solution and subsequently stained with hematoxylin and eosin (H\&E). All specimens were evaluated histopathologically by specialized pathologists who were blinded to the endoscopic diagnosis. The pathological findings were categorized as low-grade dysplasia, high-grade dysplasia (HGD) and cancer.

Statistical analysis. The paired t-test and Spearman's correlation test were used as appropriate. For all studies, p-values of $<0.05$ were set to determine the statistical significance. Statistical analysis was performed using SPSS, version 11.5, software (SPSS Inc., Chicago, IL, USA).

\section{Results}

As shown in Table I, all 11 patients had extensive colitis with the median disease duration of 14 years. The background mucosal status of all patients was in the remission stage according to the Mayo endoscopic score ( 0 or 1 point).

Clinicopathological features of the neoplastic lesion are presented in Fig. 1. All of the 15 neoplastic lesions were macroscopically identified, including 8 HGD and 7 cancer. The lesion was mostly located in the distal colon $(13 / 15,86.7 \%)$ with the mean size of $8.6 \mathrm{~mm}$. The shape was protruding in
$46.7 \%(7 / 15)$, flat elevated in $40.0 \%(6 / 15)$ and flat in $13.3 \%$ $(2 / 15)$. The color was red in $60.0 \%(9 / 15)$, same colored in $33.3 \%(5 / 15)$ and discolored in $6.7 \%(1 / 15)$.

We next analyzed the macroscopic features of the neoplastic lesions using advanced endoscopic imaging techniques. On $\mathrm{CE}$, the lesion was classified into pit patterns type $\mathrm{I}$ to $\mathrm{V}$ with type I and II representing non-neoplastic pattern and type III, IV and V representing neoplastic pattern. Of 15 lesions examined in this study, 13 lesions $(86.7 \%)$ showed neoplastic pit patterns in the order of type IV (branch- or gyrus-like crypts) $>$ type $\mathrm{III}_{\mathrm{L}}$ (large tubular or roundish crypts) $>$ type $\mathrm{V}_{\mathrm{I}}$ (irregular crypts) (Fig. 2). Two of the lesions could not be classified into pit patterns and the pattern was thus regarded as unclassified.

On NBI, the lesion was classified into type I to III capillary patterns with type I representing a non-neoplasm, type II representing adenoma and type III (subdivided into IIIa and IIIb) indicating cancer. Of the 11 lesions examined, one lesion $(0.9 \%)$ was identified as type I (faintly visible microvessels surrounding the pits), 3 lesions (27.3\%) as type II (elongated and increased thicker vessels surrounding the pits) and 7 lesions (63.6\%) as type IIIa (increased thick vessels unevenly sized with branching and curtailed irregularity) (Fig. 3).

On AFI, in general the non-neoplastic lesions appear green and neoplastic lesions appear purple. In the present study, information was available for 8 lesions from a total of 15 lesions because 7 lesions were excluded due to insufficient bowel preparation which may disrupt autofluorescence. 
A

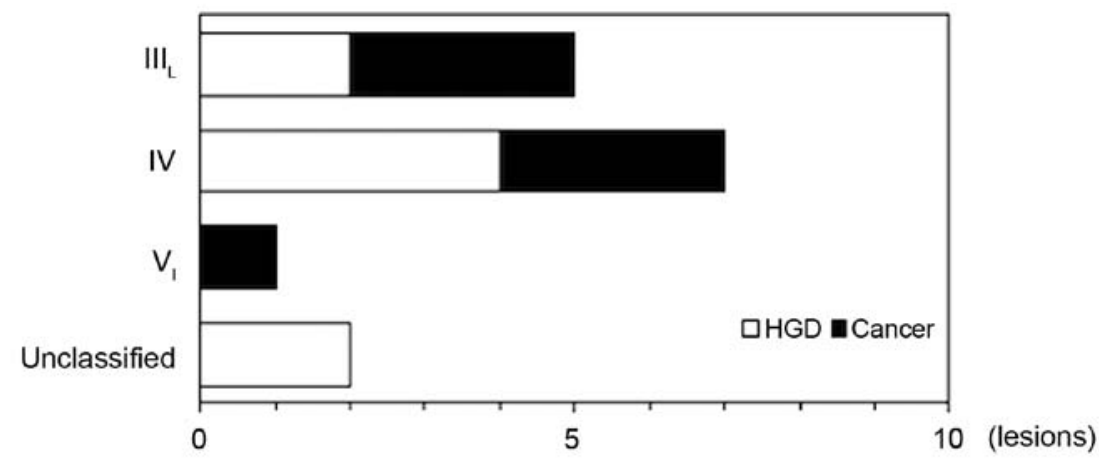

B

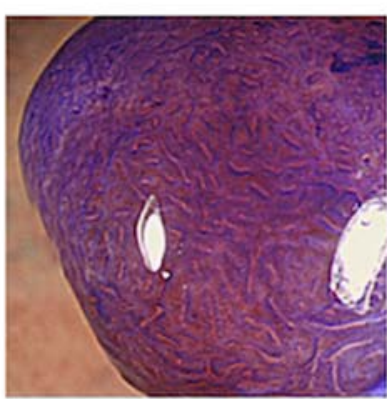

IIIL

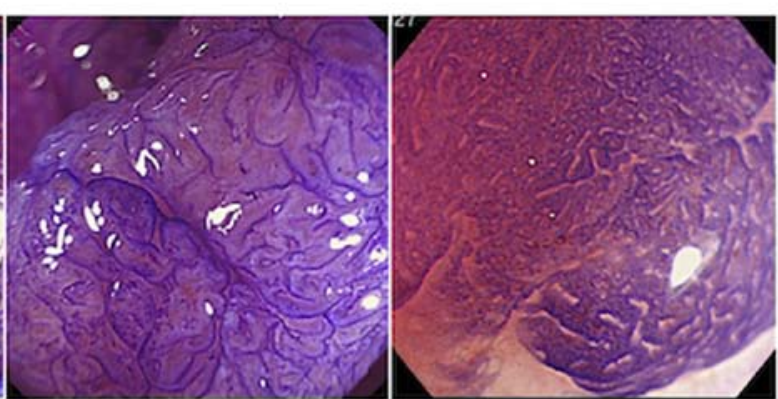

IV
$V_{1}$

Figure 2. (A) Pit pattern analysis on chromoendoscopy in colorectal neoplasia associated with UC based on the Kudo classification. Type $\mathrm{III}_{\mathrm{L}}$, large tubular or roundish crypts; type IV, branch- or gyrus-like crypts; and type $\mathrm{V}_{\mathrm{I}}$, irregular crypts $\left(\mathrm{V}_{\mathrm{I}}\right)$. HGD, high-grade dysplasia. (B) The representative image of the neoplastic lesions on chromoendoscopy with crystal violet spraying.

\section{A}

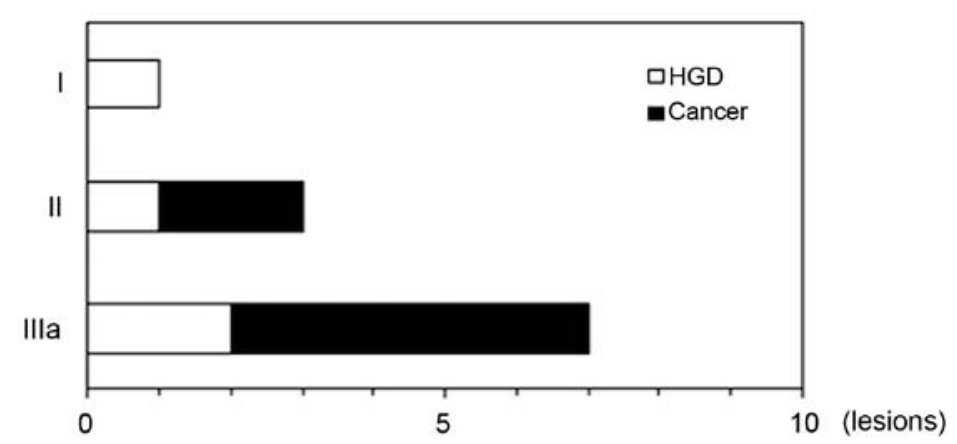

B

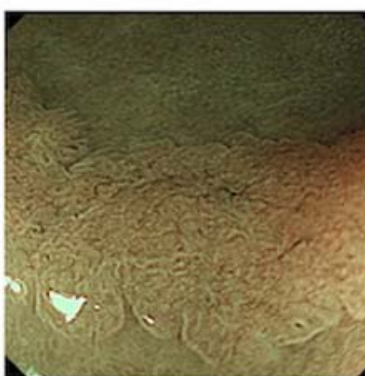

।

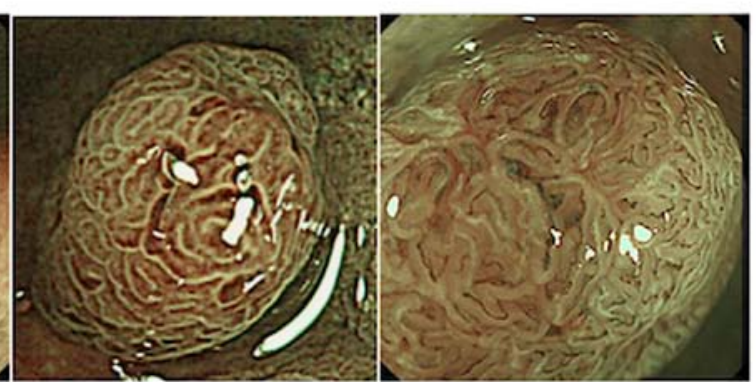

II
IIla

Figure 3. (A) Capillary pattern on narrow-band imaging of colorectal neoplasia associated with UC based on the Sano classification. Type I, faintly visible micro-vessels surrounding the pits; type II, elongated and increased thicker vessels surrounding the pits; and type IIIa, increased thick vessels unevenly sized with branching and curtailed irregularity. HGD, high-grade dysplasia. (B) The representative image of the neoplastic lesions on narrow-band imaging.

Of these, 5 lesions $(62.5 \%)$ were colored green and 3 lesions (37.5\%) colored purple (Fig. 4).

To quantify AFI intensity, the G/R ratio was determined for each lesion after adjustments for the surrounding mucosal values. Of note, the $\mathrm{G} / \mathrm{R}$ ratio was significantly lower in the neoplastic area than the adjacent non-neoplastic area involved in UC $(\mathrm{p}=0.00014)$ and the non-neoplastic area uninvolved in UC ( $\mathrm{p}=0.00651)$ (Fig. 5). The $\mathrm{G} / \mathrm{R}$ ratio was 


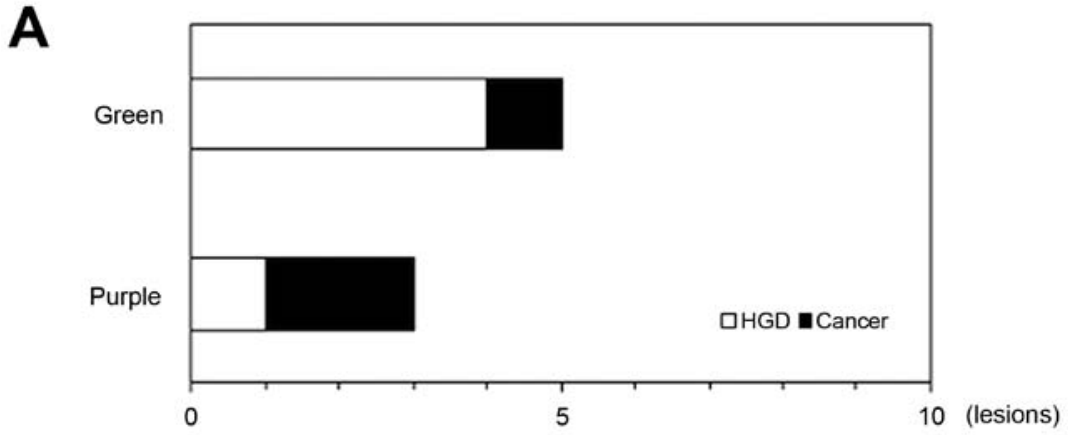

B

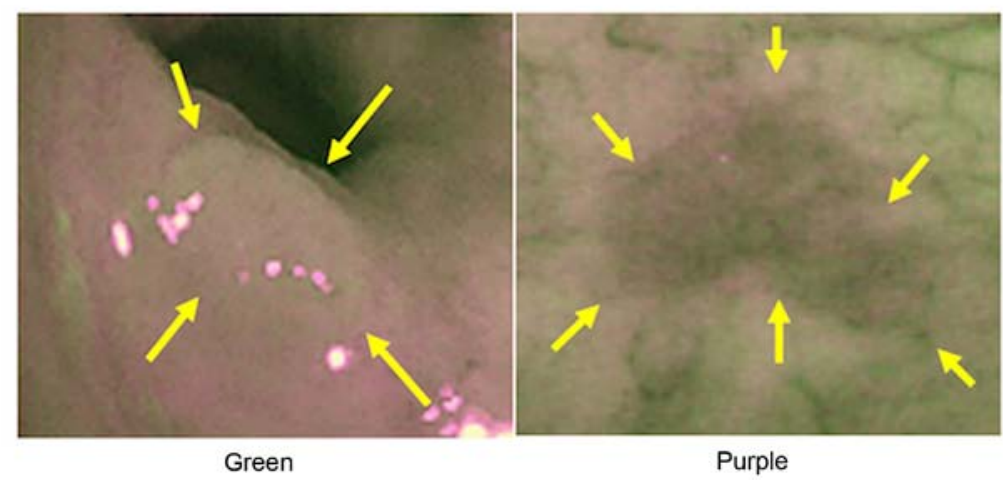

Figure 4. (A) Qualitative analysis of autofluorescence imaging (AFI) of colorectal neoplasia associated with UC. Qualitative AFI analysis is divided into green or purple. (B) A representative image of the neoplastic lesions on AFI.

not associated with the size or histological type of the lesion. Fig. 6 exemplifies the endoscopic and histological images of colorectal cancer in one patient with long-standing extensive colitis.

\section{Discussion}

Currently, several advanced endoscopic imaging techniques have been attempted to improve the limitation of conventional endoscopy (13-19). In this pilot study, we analyzed the endoscopic characteristics of neoplastic lesions associated with UC using these advanced techniques, including CE, NBI and AFI.

A better understanding of cancer risk factors may allow endoscopic resources to be more focused on patients at higher risk. It is well established that the risk for colorectal cancer increases with the duration (more than 7 years) and anatomic extent of UC (1). In line with this established observation, our study showed that neoplastic lesion tended to develop in patients with a long history of extensive colitis. Another risk, a history of primary sclerosing cholangitis, was not observed in this study probably due to the small number of the patients in this study.

The neoplastic lesions in our study were predominantly located in the distal colon $(86.7 \%)$, appeared protruded $(46.7 \%)$ or flat elevated $(40.0 \%)$, and were colored red $(60.0 \%)$. These findings are consistent with previous reports describing how dysplasia and early cancer were characterized by low protruding or flat mucosa, often associated with redness (29-33).

$\mathrm{CE}$ is used to better define the superficial mucosa. CE with dye agents enhances the mucosal detail and permits a more precise characterization. Kudo et al classified the pit patterns into types I to V $(24,25)$. As reported by Sada et al (29), some neoplastic lesions associated with UC have a neoplastic type $\mathrm{III}_{\mathrm{S}}$ to $\mathrm{III}_{\mathrm{L}}$ or type IV-type pit patterns. In contrast, Hata et al $(30,31)$ reported that type III or IV pit patterns were not observed in some dysplastic lesions. Our study showed that 13 of the 15 dysplasia/cancer lesions (86.7\%) were representative of the neoplastic pit pattern, suggesting that $\mathrm{CE}$ is helpful to detect and discriminate neoplastic lesions in UC although coexisting inflammatory changes may modify the mucosal detail.

NBI is an endoscopy system, which enables a clear visualization of the microvasculature of colorectal lesions $(26,27)$. Previous investigations have shown that NBI is effective in distinguishing neoplastic colorectal lesions in sporadic settings $(16,17)$. Our present study showed that 7 of the 11 dysplasia/cancer lesions $(63.6 \%)$ were representative of the neoplastic capillary pattern. Together with the results from recent randomized trials demonstrating that NBI was a reasonable alternative to CE (34-37), NBI is one of the available modalities to distinguish neoplastic lesion associated with UC.

AFI is a novel technique that is based on the fact that tissues exhibit fluorescence when exposed to ultraviolet ( $<400 \mathrm{~nm}$ ) or shorter waveband visible light (mostly blue) (19). Recent investigations have demonstrated the usefulness of AFI in discriminating colorectal neoplasia in sporadic setting (18). However, the available data on AFI for UC surveillance is sparse.

On AFI, neoplastic tissue is visible as a purple lesion on a green background fluorescence of normal colonic tissue $(18,19)$. Our study showed that a purple lesion was observed only in $37.5 \%$ of the neoplastic lesions, indicating that the qualitative analysis of the AFI color, green or purple, is not so helpful for detecting neoplasia associated with UC. 

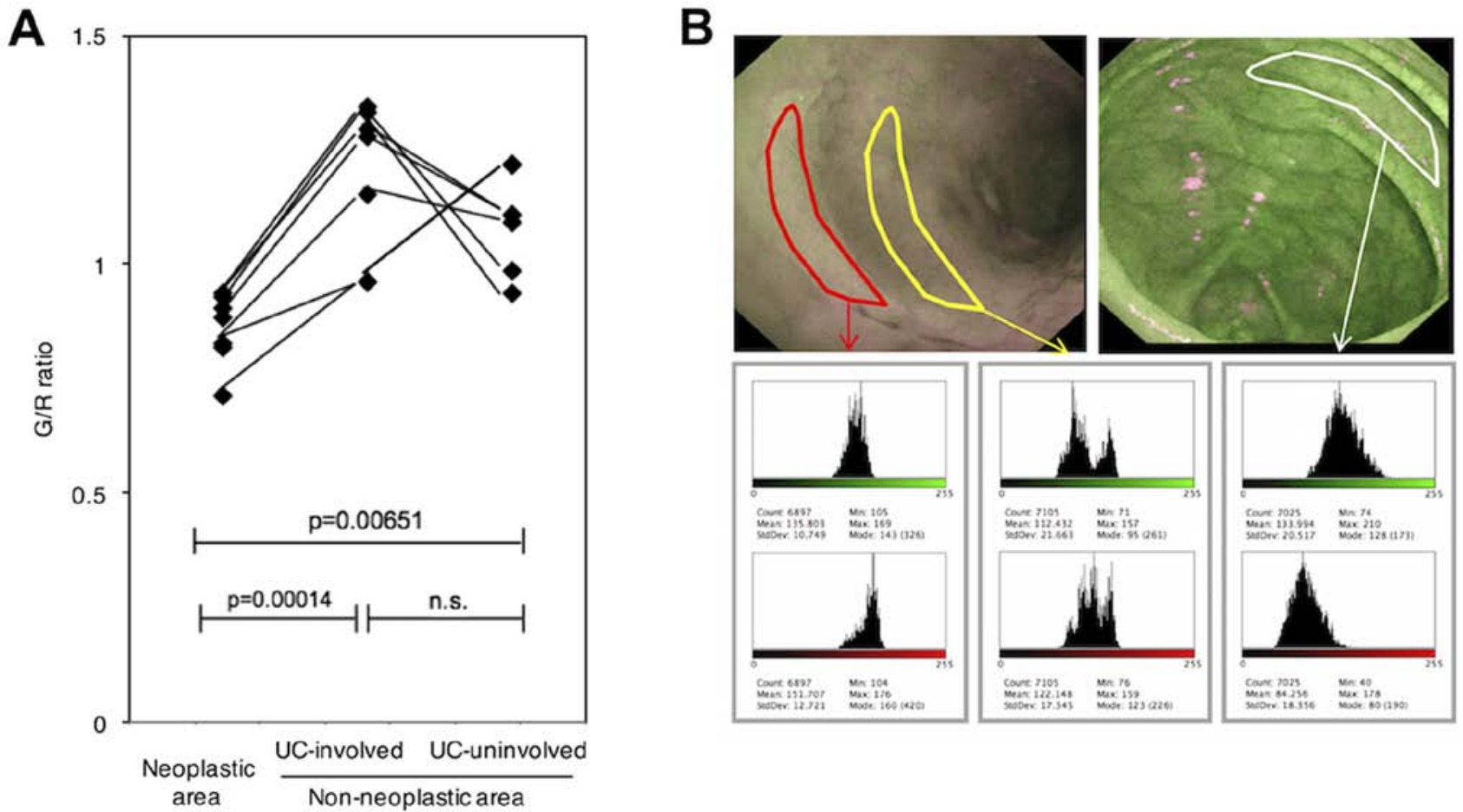

Figure 5. (A) Quantitative analysis of autofluorescence imaging (AFI) of colorectal neoplasia associated with UC using the green-to-red (G/R) ratio. The G/R ratio was measured in the neoplastic area, the adjacent non-neoplastic area involved in UC, and the non-neoplastic area uninvolved in UC. (B) The representative AFI image of the neoplastic area (red box) and the adjacent non-neoplastic area involved in UC (yellow box), and the non-neoplastic area uninvolved in UC (white box).

A

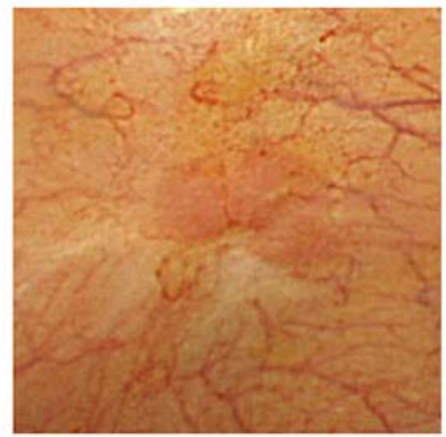

B

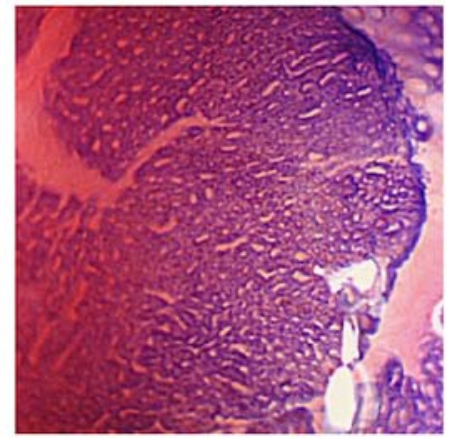

C
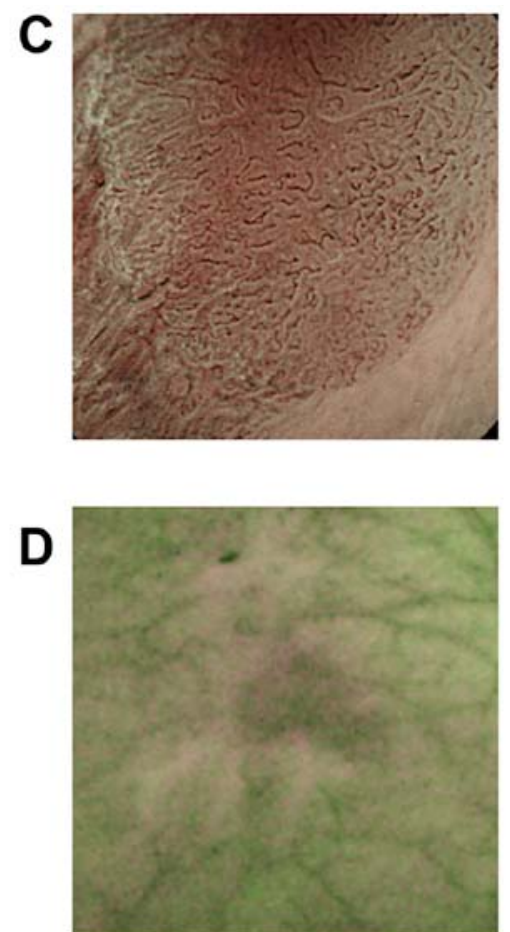

E

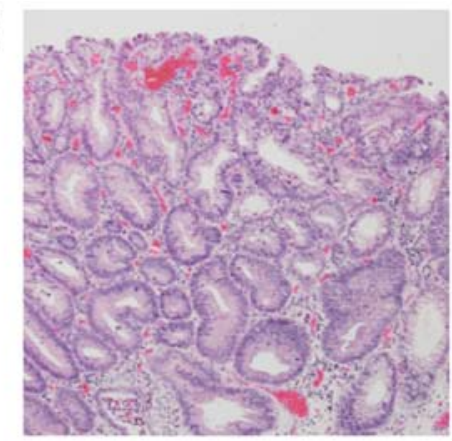

$\mathbf{F}$

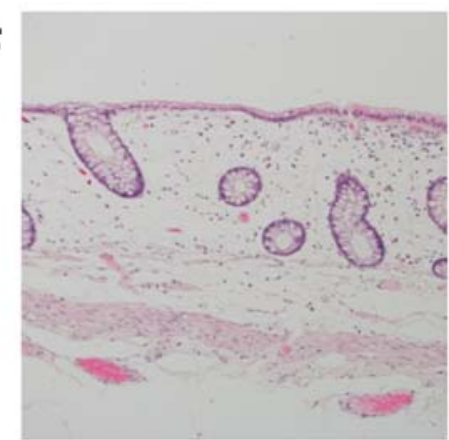

Figure 6. Representative endoscopic and histological images of colorectal cancer in one patient with long-standing extensive colitis. A flat elevated lesion on white-light imaging (A) revealed pit pattern type $\mathrm{V}_{\mathrm{I}}$ on chromoendoscopy with crystal violet spraying (B) and capillary pattern type III on narrow-band imaging (C) and appeared purple on autofluorescence imaging (D). Well-differentiated adenocarcinoma in the elevated lesion (E) and the adjacent quiescent area of chronic injury (F) were histologically observed. H\&E; original magnification, x20.

One possible explanation is that the AFI color is altered according to the grade of inflammation $(38,39)$. This is probably due to several factors that modify the AFI color both in inflammation and neoplasia, such as tissue architecture, light absorption and 
scattering properties, the biochemical content, or metabolic status of the tissue $(40,41)$.

Inomata et al demonstrated that quantitative analysis of the AFI color using the $\mathrm{G} / \mathrm{R}$ ratio was effective in distinguishing sporadic neoplastic lesions (28). The most important observation in the present study is that we found for the first time that the $\mathrm{G} / \mathrm{R}$ ratio is helpful in discriminating dysplasia/cancer in $\mathrm{UC}$ as well. The $\mathrm{G} / \mathrm{R}$ ratio was not associated with the lesion size or histological type, supporting the idea that this index is available even for smaller lesions. Quantitative AFI, rather than qualitative, has therefore the potential for detection of early neoplastic changes in UC. The field of AFI is still young and multiple questions remain unanswered. However, our results in AFF quantification are very promising but as we gain more experience, these will be better defined, hence the need for further investigations.

There are several limitations to our study. First, this was a single-center analysis involving only a limited number of patients with a retrospective design. Second, because of a retrospective design, the diagnosis in the study subjects had been established. Although advanced endoscopic imaging such as CE, NBI and AFI look very promising, large-scale, prospective study will be needed to assess their place in the surveillance of UC patients.

In conclusion, this pilot study suggested that endoscopic analysis based on advanced imaging, in particular AFI quantitation, is helpful to detect early stage neoplastic lesions in long standing UC.

\section{Acknowledgements}

The present study was supported in part by a Grant-in-Aid from the Japanese Ministry of Education, Culture and Science (no. 25460964) and Health and Labour Sciences Research Grants for research on intractable diseases from the Ministry of Health, Labour and Welfare of Japan.

\section{References}

1. Eaden JA, Abrams KR and Mayberry JF: The risk of colorectal cancer in ulcerative colitis: A meta-analysis. Gut 48: 526-535, 2001.

2. Ullman TA and Itzkowitz SH: Intestinal inflammation and cancer. Gastroenterology 140: 1807-1816, 2011.

3. Morson BC and Pang LS: Rectal biopsy as an aid to cancer control in ulcerative colitis. Gut 8: 423-434, 1967.

4. Bernstein CN, Shanahan F and Weinstein WM: Are we telling patients the truth about surveillance colonoscopy in ulcerative colitis? Lancet 343: 71-74, 1994.

5. Blackstone MO, Riddell RH, Rogers BH and Levin B: Dysplasiaassociated lesion or mass (DALM) detected by colonoscopy in long-standing ulcerative colitis: An indication for colectomy. Gastroenterology 80: 366-374, 1981 .

6. Farrell RJ and Peppercorn MA: Ulcerative colitis. Lancet 359: 331-340, 2002

7. Eaden JA and Mayberry JF; British Society for Gastroenterology Association of Coloproctology for Great Britain and Ireland: Guidelines for screening and surveillance of asymptomatic colorectal cancer in patients with inflammatory bowel disease Gut 51 (Suppl 5): V10-V12, 2002.

8. Winawer S, Fletcher R, Rex D, Bond J, Burt R, Ferrucci J, Ganiats T, Levin T, Woolf S, Johnson D, et al; Gastrointestinal Consortium Panel: Colorectal cancer screening and surveillance: Clinical guidelines and rationale - Update based on new evidence. Gastroenterology 124: 544-560, 2003.

9. Kornbluth A and Sachar DB: Ulcerative colitis practice guidelines in adults. American College of Gastroenterology, Practice Parameters Committee. Am J Gastroenterol 92: 204-211, 1997.
10. Rutter MD: Surveillance programmes for neoplasia in colitis. J Gastroenterol 46 (Suppl 1): 1-5, 2011.

11. Watanabe $\mathrm{T}$, Ajioka $\mathrm{Y}$, Matsumoto $\mathrm{T}$, Tomotsugu $\mathrm{N}$, Takebayashi T, Inoue E, Iizuka B, Igarashi M, Iwao Y, Ohtsuka K, et al: Target biopsy or step biopsy? Optimal surveillance for ulcerative colitis: A Japanese nationwide randomized controlled trial. J Gastroenterol 46 (Suppl 1): 11-16, 2011.

12. Matsumoto T, Iwao Y, Igarashi M, Watanabe K, Otsuka K, Watanabe T, Iizuka B, Hida N, Sada M, Chiba T, et al: Endoscopic and chromoendoscopic atlas featuring dysplastic lesions in surveillance colonoscopy for patients with long-standing ulcerative colitis. Inflamm Bowel Dis 14: 259-264, 2008.

13. Basu S, Torigian D and Alavi A: The role of modern molecular imaging techniques in gastroenterology. Gastroenterology 135: 1055-1061, 2008.

14. Axelrad AM, Fleischer DE, Geller AJ, Nguyen CC, Lewis JH, Al-Kawas FH, Avigan MI, Montgomery EA and Benjamin SB: High-resolution chromoendoscopy for the diagnosis of diminutive colon polyps: Implications for colon cancer screening. Gastroenterology 110: 1253-1258, 1996.

15. Fu KI, Sano Y, Kato S, Fujii T, Nagashima F, Yoshino T, Okuno T, Yoshida S and Fujimori T: Chromoendoscopy using indigo carmine dye spraying with magnifying observation is the most reliable method for differential diagnosis between nonneoplastic and neoplastic colorectal lesions: A prospective study. Endoscopy 36: 1089-1093, 2004.

16. Sano Y, Muto M, Tajiri H, Ohtsu A and Yoshida S: Optical/digital chromoendoscopy during colonoscopy using narrow-band image system. Dig Endosc 17 (Suppl 1): S43-S48, 2005. http://onlinelibrary. wiley.com/doi/10.1111/j.1443-1661.2005.00511.x/abstract.

17. Chiu HM, Chang CY, Chen CC, Lee YC, Wu MS, Lin JT, Shun CT and Wang HP: A prospective comparative study of narrow-band imaging, chromoendoscopy, and conventional colonoscopy in the diagnosis of colorectal neoplasia. Gut 56: 373-379, 2007.

18. Arita K, Mitsuyama K, Kawano H, Hasegawa S, Maeyama Y, Masuda J, Akagi Y, Watanabe Y, Okabe Y, Tsuruta O, et al: Quantitative analysis of colorectal mucosal lesions by autofluorescence endoscopy: Discrimination of carcinomas from other lesions. Oncol Rep 26: 43-48, 2011.

19. Panjehpour M, Overholt BF, Vo-Dinh T, Haggitt RC, Edwards DH and Buckley FP III: Endoscopic fluorescence detection of highgrade dysplasia in Barrett's esophagus. Gastroenterology 111: 93-101, 1996.

20. Gabbani T, Manetti N, Bonanomi AG, Annese AL and Annese V: New endoscopic imaging techniques in surveillance of inflammatory bowel disease. World J Gastrointest Endosc 7: 230-236, 2015.

21. Subramanian V and Bisschops R: Image-enhanced endoscopy is critical in the surveillance of patients with colonic IBD. Gastrointest Endosc Clin N Am 24: 393-403, 2014.

22. Efthymiou M, Taylor AC and Kamm MA: Cancer surveillance strategies in ulcerative colitis: The need for modernization. Inflamm Bowel Dis 17: 1800-1813, 2011.

23. Hasegawa S, Mitsuyama K, Kawano H, Arita K, Maeyama Y, Akagi Y, Watanabe Y, Okabe Y, Tsuruta $O$ and Sata M: Endoscopic discrimination of sessile serrated adenomas from other serrated lesions. Oncol Lett 2: 785-789, 2011.

24. Kudo S, Hirota S, Nakajima T, Hosobe S, Kusaka H, Kobayashi T, Himori $\mathrm{M}$ and Yagyuu A: Colorectal tumours and pit pattern. J Clin Pathol 47: 880-885, 1994.

25. Kudo S, Tamura S, Nakajima T, Yamano H, Kusaka H and Watanabe H: Diagnosis of colorectal tumorous lesions by magnifying endoscopy. Gastrointest Endosc 44: 8-14, 1996.

26. Uraoka T, Saito Y, Ikematsu H, Yamamoto K, Sano Y: Sano's capillary pattern classification for narrow-band imaging of early colorectal lesions. Dig Endosc 23 (Suppl 1): 112-115, 2011. http:// dx.doi.org/10.1111/j.1443-1661.2011.01118.x.

27. Sakamoto T, Saito Y, Nakajima T, Matsuda T: Comparison of magnifying chromoendoscopy and narrow-band imaging in estimation of early colorectal cancer invasion depth: a pilot study. Dig Endosc 23: 118-123, 2011. http://dx.doi.org/10.1111/j.14431661.2010.01049.x

28. Inomata $\mathrm{H}$, Tamai $\mathrm{N}$, Aihara $\mathrm{H}$, Sumiyama $\mathrm{K}$, Saito $\mathrm{S}$, Kato $\mathrm{T}$ and Tajiri H: Efficacy of a novel auto-fluorescence imaging system with computer-assisted color analysis for assessment of colorectal lesions. World J Gastroenterol 19: 7146-7153, 2013.

29. Sada M, Igarashi M, Yoshizawa S, Kobayashi K, Katsumata T, Saigenji K, Otani Y, Okayasu I and Mitomi H: Dye spraying and magnifying endoscopy for dysplasia and cancer surveillance in ulcerative colitis. Dis Colon Rectum 47: 1816-1823, 2004. 
30. Hata K, Watanabe T, Motoi T and Nagawa H: Pitfalls of pit pattern diagnosis in ulcerative colitis-associated dysplasia. Gastroenterology 126: 374-376, 2004.

31. Hata K, Watanabe T, Kazama S, Suzuki K, Shinozaki M, Yokoyama T, Matsuda K, Muto T and Nagawa H: Earlier surveillance colonoscopy programme improves survival in patients with ulcerative colitis associated colorectal cancer: Results of a 23-year surveillance programme in the Japanese population. Br J Cancer 89: 1232-1236, 2003.

32. Hata K, Watanabe T, Shinozaki M, Kojima T and Nagawa $H$ : To dye or not to dye? That is beyond question! Optimising surveillance colonoscopy is indispensable for detecting dysplasia in ulcerative colitis. Gut 53: 1722, 2004.

33. Matsumoto T, Nakamura S, Jo Y, Yao T and Iida M: Chromoscopy might improve diagnostic accuracy in cancer surveillance for ulcerative colitis. Am J Gastroenterol 98: 1827-1833, 2003.

34. Pellisé M, López-Cerón M, Rodríguez de Miguel C, Jimeno M, Zabalza M, Ricart E, Aceituno M, Fernández-Esparrach G, Ginès A, Sendino O, et al: Narrow-band imaging as an alternative to chromoendoscopy for the detection of dysplasia in long-standing inflammatory bowel disease: A prospective, randomized, crossover study. Gastrointest Endosc 74: 840-848, 2011.

35. Günther U, Kusch D, Heller F, Bürgel N, Leonhardt S, Daum S, Siegmund B, Loddenkemper C, Grünbaum M, Buhr HJ, et al: Surveillance colonoscopy in patients with inflammatory bowel disease: Comparison of random biopsy vs. targeted biopsy protocols. Int J Colorectal Dis 26: 667-672, 2011.
36. Kiesslich R, Goetz M, Lammersdorf K, Schneider C, Burg J, Stolte M, Vieth M, Nafe B, Galle PR and Neurath MF: Chromoscopy-guided endomicroscopy increases the diagnostic yield of intraepithelial neoplasia in ulcerative colitis. Gastroenterology 132: 874-882, 2007.

37. Kiesslich R, Fritsch J, Holtmann M, Koehler HH, Stolte M, Kanzler S, Nafe B, Jung M, Galle PR and Neurath MF: Methylene blue-aided chromoendoscopy for the detection of intraepithelial neoplasia and colon cancer in ulcerative colitis. Gastroenterology 124: 880-888, 2003.

38. Rubin DT and Turner JR: Surveillance of dysplasia in inflammatory bowel disease: The gastroenterologist-pathologist partnership. Clin Gastroenterol Hepatol 4: 1309-1313, 2006. http://europepmc.org/abstract/MED/17110299.

39. Matsumoto T, Moriyama T, Yao T, Mibu R and Iida M: Autofluorescence imaging colonoscopy for the diagnosis of dysplasia in ulcerative colitis. Inflamm Bowel Dis 13: 640-641, 2007.

40. Zonios GI, Cothren RM, Arendt JT, Wu J, Van Dam J, Crawford JM, Manoharan R and Feld MS: Morphological model of human colon tissue fluorescence. IEEE Trans Biomed Eng 43: 113-122, 1996.

41. Marchesini R, Pignoli E, Tomatis S, Fumagalli S, Sichirollo AE, Di Palma S, Dal Fante M, Spinelli P, Croce AC and Bottiroli G: Ex vivo optical properties of human colon tissue. Lasers Surg Med 15: 351-357, 1994. 